\title{
Acute effects of vagus nerve stimulation parameters on gastric motility assessed with magnetic resonance imaging
}

\author{
Kun-Han Lu', (D) | Jiayue Cao ${ }^{3}$ | Robert Phillips ${ }^{4}$ | Terry L. Powley ${ }^{2,4}$ (D) | \\ Zhongming Liu ${ }^{3,5}$ (1)
}

${ }^{1}$ Weldon School of Biomedical Engineering, Purdue University, West Lafayette, IN, USA

${ }^{2}$ Purdue Institute for Integrative

Neuroscience, Purdue University, West

Lafayette, IN, USA

${ }^{3}$ Biomedical Engineering, University of Michigan, Ann Arbor, MI, USA

${ }^{4}$ Department of Psychological Sciences,

Purdue University, West Lafayette, IN, USA

${ }^{5}$ Electrical Engineering and Computer Science, University of Michigan, Ann Arbor, MI, USA

\section{Correspondence}

Kun-Han Lu, Weldon School of Biomedical Engineering, College of Engineering, Purdue University, 206 S. Martin Jischke Dr., West Lafayette, IN 47907, USA.

Email: lu415@purdue.edu

Funding information

This work was supported in part by $\mathrm{NIH}$ SPARC 1OT2TR001965 and Purdue University.

\begin{abstract}
Background: Vagus nerve stimulation (VNS) is an emerging bioelectronic therapy for regulating food intake and controlling gastric motility. However, the effects of different VNS parameters and polarity on postprandial gastric motility remain incompletely characterized.

Methods: In anesthetized rats ( $\mathrm{N}=3$ ), we applied monophasic electrical stimuli to the left cervical vagus and recorded compound nerve action potential (CNAP) as a measure of nerve response. We evaluated to what extent afferent or efferent pathway could be selectively activated by monophasic VNS. In a different group of rats ( $N=13$ ), we fed each rat a gadolinium-labeled meal and scanned the rat stomach with oral contrast-enhanced magnetic resonance imaging (MRI) while the rat was anesthetized. We evaluated the antral and pyloric motility as a function of pulse amplitude $(0.13,0.25,0.5,1 \mathrm{~mA})$, width $(0.13,0.25,0.5 \mathrm{~ms})$, frequency $(5,10 \mathrm{~Hz})$, and polarity of VNS.

Key Results: Monophasic VNS activated efferent and afferent pathways with about $67 \%$ and $82 \%$ selectivity, respectively. Primarily afferent VNS increased antral motility across a wide range of parameters. Primarily efferent VNS induced a significant decrease in antral motility as the stimulus intensity increased $(R=-.93, P<.05$ for $5 \mathrm{~Hz}, R=-.85, P<.05$ for $10 \mathrm{~Hz}$ ). The VNS with either polarity tended to promote pyloric motility to a greater extent given increasing stimulus intensity.

Conclusions and Inferences: Monophasic VNS biased toward the afferent pathway is potentially more effective for facilitating occlusive contractions than that biased toward the efferent pathway.
\end{abstract}

KEYWORDS

gastric motility, magnetic resonance imaging, rat, vagus nerve stimulation

\section{1 | INTRODUCTION}

The parasympathetic innervation to the gastrointestinal (GI) tract plays a key role in regulating, modulating, and controlling GI motility and maintaining energy homeostasis. ${ }^{1,2}$ Because this innervation is predominantly supplied by the vagus nerve, ${ }^{3,4}$ it has been of great interest to modulate motor neural signaling to the gut via electrical stimulation of the vagus nerve. ${ }^{5-7}$ However, several morphological and functional studies have suggested that the vagus is a heterogeneous nerve consisting of distinct fiber calibers that carries both efferent and afferent traffic. ${ }^{8,9}$ The stimulus-response relations often vary according to the site of stimulation and also the choice of stimulus parameters. As 
a result, the complete extent of vagal innervation to the enteric neural plexuses remains incompletely characterized.

The rat vagus is comprised of about $75 \%$ sensory afferent and $25 \%$ motor efferent fibers. ${ }^{8}$ Previous preclinical studies have shown that electrical stimulation of the vagus could impose either excitatory or relaxatory effect on gastric tone and motility. ${ }^{10-13}$ While the stimulation effect was reported to be dependent on stimulus parameters, another potentially critical factor that may alter physiological response is the polarity of stimulation. In conventional bipolar electrode setting, the polarity of stimulation is determined by the placement of anode and cathode along the nerve. According to the principle of anodal blocking effect, ${ }^{14}$ the nerve tissue at the cathode depolarizes and evokes compound nerve action potential (CNAP), whereas the nerve tissue at the anode hyperpolarizes and may block, at least in part if not entirely, the propagation of CNAP. Therefore, placing the cathode rostral to the anode could be biased for nerve activation in the afferent pathway (hereinafter referred to as afferent VNS for simplicity). On the other hand, placing the cathode caudal to the anode could be biased for nerve activation in the efferent pathway (hereinafter referred to as efferent VNS for simplicity). As such, elucidating the potentially differential impact of stimulus polarity on gastric physiology is necessary for better calibration of neuromodulation efficacy.

To evaluate the efficacy and stability of stimulus parameters, most studies employed invasive methods in fasted animals. However, it is possible that the stimulus-response relations might be confounded by unphysiological assessments. Moreover, as electroceutical therapeutic outcomes in humans are typically derived during meal digestion, the importance of characterizing the stimulation effect under postprandial state should be noted. To address this need, we have recently developed an oral contrast-enhanced magnetic resonance imaging (MRI) protocol to non-invasively monitor gastric emptying and motility in DietGel-fed rats. ${ }^{15,16}$

The purpose of this study was to examine the impact of left cervical VNS with different parameters and stimulus polarity on postprandial gastric motility in anesthetized rats. Oral contrast-enhanced gastric MRI was performed continuously before and during VNS, where the occlusive amplitude of antral contraction and the tone of pyloric opening were measured as a function of VNS settings. We hypothesize that (a) the polarity of stimulation may potentially alter gastric response to VNS, and (b) as the stimulation intensity increases, vagal fibers with higher threshold (eg, unmyelinated $C$ fibers) would be increasingly recruited, which may lead to differential gastric response compared to the response elicited by low stimulation intensity.

\section{2 | MATERIALS AND METHODS}

\section{1 | Subjects}

Sixteen Sprague Dawley rats (Male; Envigo RMS) were used in this study. All experimental procedures were approved by the Purdue

\section{Key Points}

- Vagus nerve stimulation (VNS) is emerging as a new bioelectronic therapy for remedying gastric symptoms. However, the effects of graded VNS parameters and polarity preferences of VNS on gastric functions remain incompletely characterized.

- Oral contrast-enhanced gastric MRI revealed differential effects of primarily afferent versus efferent VNS on antral and pyloric motility.

- MRI can non-invasively characterize postprandial gastric motility with high spatial and temporal resolution that could be used to guide and refine the selection of VNS parameters in preclinical settings.

Animal Care and Use Committee (PACUC) and the Laboratory Animal Program (LAP). The animals were housed individually in ventilated cages under a strictly controlled environment (temperature: $70 \pm 2^{\circ} \mathrm{F}$, and 12 hours light-dark cycle: lights on at 6:00 AM, lights off at 6:00 PM). The floor was elevated by a stainless-steel wireframe during all time to prevent the animals from accessing their feces. All experiments were performed acutely, after which the animals were euthanized according to a standard approved protocol.

\section{2 | Test meal}

All animals were trained to voluntarily consume a fixed quantity (about $10 \mathrm{~g}$ ) of nutrient-fortified water gel (DietGel Recovery, ClearH2O) under a re-feeding condition following an overnight (18 hours, 5 PM to 11 AM) fast. The training protocol consisted of 2 stages. During the first stage, an aliquot $(\sim 10 \mathrm{~g})$ of the DietGel was placed in a cup in animal's home cage overnight for 2 times, with the regular rat chow being supplied ad libitum. Once the animal got used to the test meal, their food was deprived for 18 hours followed by re-feeding of the DietGel. At the end of the 7-day training, all animals were compliant to the feeding paradigm in order for us to investigate gastric functions in a physiologically fed condition.

\subsection{Animal preparation and surgical implantation of stimulation electrode}

On the day of imaging, the animal was fed with $10 \mathrm{~g}$ of gadolinium (Gd)-labeled test meal after the 18-hour overnight fast. The Gdlabeled test meal was made of $10 \mathrm{~g}$ DietGel mixed with $22.4 \mathrm{mg}$ Gd-DTPA powder (\#381667, Sigma-Aldrich) using a double-boiled liquefying approach. Immediately after it had ingested the meal, the animal was anesthetized with isoflurane followed by implantation of a bipolar cuff electrode (Pt/Ir electrode; MicroProbes) onto 
the left cervical vagus nerve with a surgical procedure identical to that described in our previous work. ${ }^{16}$ The animal was then set up in prone position in the scanner. Respiration and body temperature were monitored with an MR-compatible system (SA Instruments Inc) to ensure stable physiology. The leads of the electrode were connected to a pair of twisted wires that were connected to a current stimulator (A-M Systems Model 2200; A-M Systems) placed outside of the MRI room. A bolus injection of $0.01 \mathrm{mg} \mathrm{kg}^{-1}$ dexmedetomidine solution (0.05 $\mathrm{mg} \mathrm{mL}^{-1}$, Zoetis) was administered subcutaneously (SC). Fifteen minutes after the bolus, dexmedetomidine was infused (SC) continuously throughout the experiment $\left(0.03 \mathrm{mg} \mathrm{kg}^{-1} \mathrm{~h}^{-1}\right)$. The dose of Isoflurane was lowered to $0.3 \%-0.5 \%$ as soon as the animal's respiratory rate began to decrease.

\section{4 | MRI data acquisition}

Dynamic gastric MRI images were acquired with a 7-Tesla horizontalbore small-animal system (BioSpec 70/30, Bruker) and an imaging protocol adopted from our previous study. ${ }^{15}$ Briefly, an abdominal localizer was first applied to reveal the long axis of the stomach from $\mathrm{T}_{2}$-weighted sagittal images. Then, a fast low angle shot gradient echo (FLASH) sequence with 4 slices was prescribed along the long axis of the stomach. The 4 slices were carefully positioned and adjusted to cover the antrum, pylorus, and the duodenum. The MRI scans were acquired with $\mathrm{TR} / \mathrm{TE}=11.78 / 1.09 \mathrm{~ms}, \mathrm{FA}=25^{\circ}, 4$ oblique slices, slice thickness $=1.5 \mathrm{~mm}, \mathrm{FOV}=60 \times 60 \mathrm{~mm}^{2}$, in-plane resolution $=0.4688 \times 0.4688 \mathrm{~mm}^{2}$, and no averaging. To reduce motion artifact in the images, the MRI scans were respiratory-gated such that images were acquired only during the end-expiratory phase with minimum diaphragmatic motion. Readout gradient triggers were recorded to adjust acquisition times between volumes due to varying respiratory rates throughout the experiment. Depending on the respiratory pattern of the animal, the resulting sampling rate was in general between 2 to 3 seconds per volume, which was sufficient to capture antral peristalsis in rats (about 5-6 cycles per minute).

\section{5 | Experimental protocol}

After obtaining about 5 minutes of stable, baseline dynamic MRI images, cervical VNS was delivered simultaneously with MRI acquisition. Of the 16 rats, 13 rats (body weight: $266-338 \mathrm{~g}$ ) were allocated into 2 groups as follows: (a) a group of rats $(\mathrm{N}=8)$ that received afferent VNS (ie, cathode placed rostral to the anode) and (b) a group of rats $(N=5)$ that received efferent VNS (ie, cathode placed caudal to the anode), as illustrated in Figure 1A,B. In addition to evaluating the effect of afferent vs efferent VNS on gastric motility, we further applied bidirectional VNS (ie, combined afferent and efferent VNS) to the rats in the afferent VNS group, immediately after which afferent VNS was performed. The bidirectional VNS was achieved by delivering monophasic pulses of current in alternating directions between the two electrodes as elaborated elsewhere. ${ }^{16}$

For each VNS group, the stimulus parameters were varied in terms of pulse amplitude (PA: 0.13, 0.25, 0.5, $1 \mathrm{~mA}$ ), width (PW: $0.13,0.25,0.5 \mathrm{~ms}$ ), and frequency (PF: $5,10 \mathrm{~Hz}$ ). The frequency of afferent or efferent VNS was defined as the number of electrical pulses delivered per second. The frequency of bidirectional VNS was defined as the number of paired cathodal and anodal pulses delivered per second. Low, medium, and high values were selected for each parameter settings, all of which are frequently used in clinical and preclinical settings. Here, a stimulus dose was defined as the area under individual electrical pulse (product of pulse amplitude $[\mathrm{mA}]$ and pulse width $[\mathrm{ms}]$ ), representing the electrical charge (Q; micro coulomb $[\mu \mathrm{C}]$ ) delivered to the nerve tissue. For bidirectional VNS, the stimulus dose for a pair of cathodal and anodal pulses was defined as the charge under the cathodal pulse, because the effect of afferent vs efferent VNS was treated
FIGURE 1 Illustration of experimental design. (A) Experimental protocol. (B) The polarity of vagus nerve stimulation (VNS) was controlled by the placement of the anode (+) and cathode (-) of the bipolar cuff electrode along the nerve. (C) Dynamic gastric MRI images were collected before and during VNS. Every VNS setting was performed for 1 minute followed by another minute of rest. The sequence of different VNS settings was randomized within and across animals. CNAP, Compound nerve action potential

\section{(A) Experimental protocol}

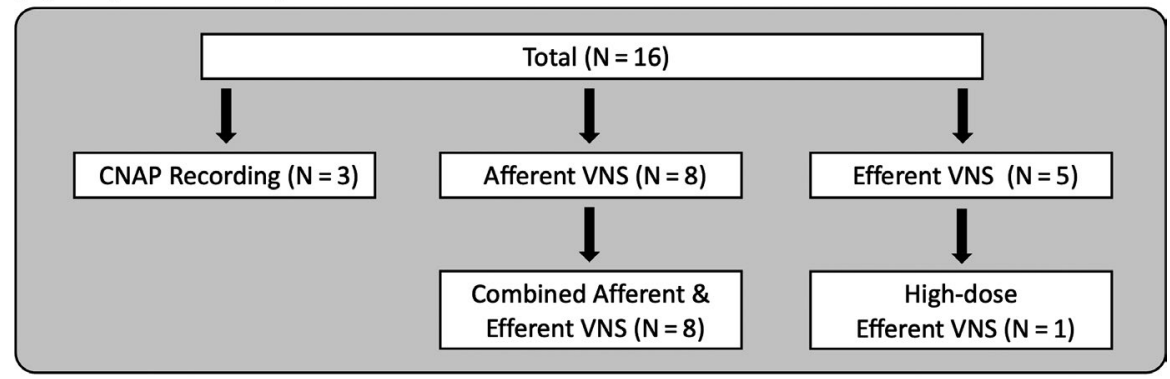

(B) Polarity of VNS

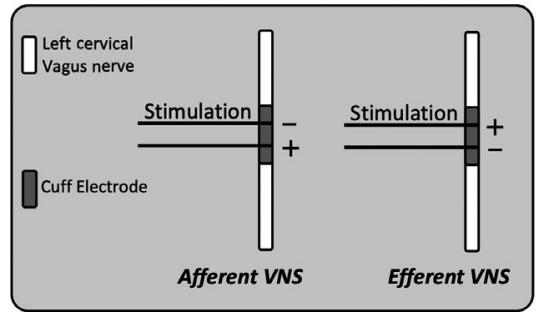

(C) Experimental Paradigm

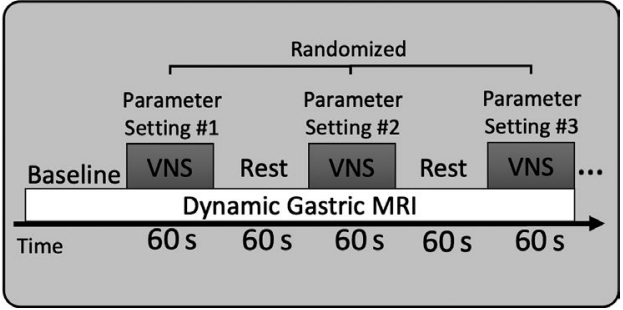




\section{(A) Stimulation (Rostral) - Recording (Caudal)}
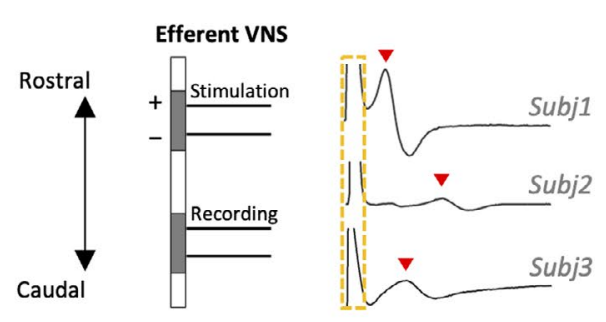
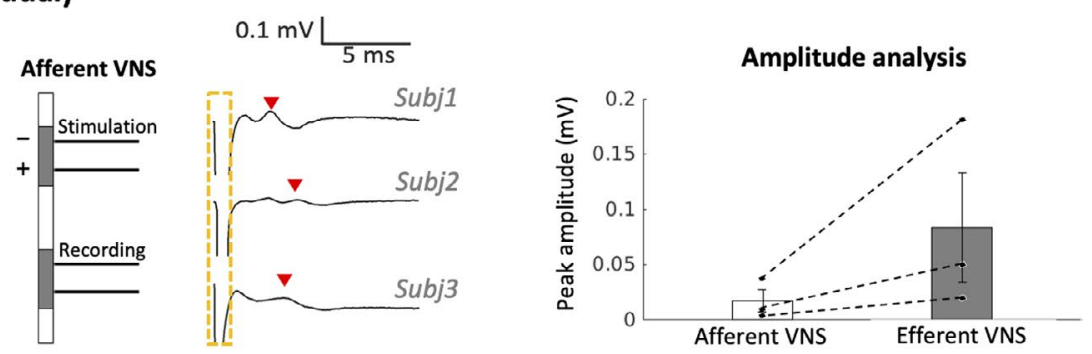

(B) Stimulation (Caudal) - Recording (Rostral)
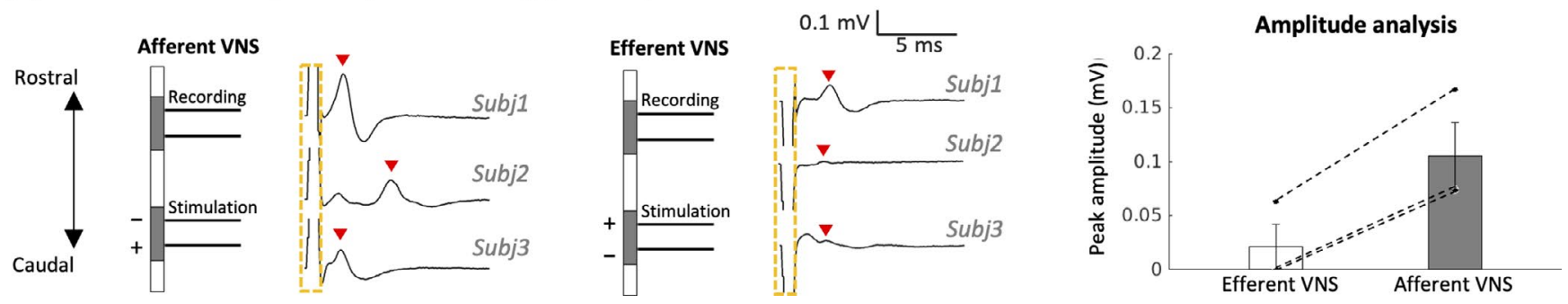

FIGURE 2 Recording and analysis of compound nerve action potential (CNAP). To record CNAP, a bipolar recording cuff electrode was placed about $3 \mathrm{~mm}$ below (A) or above (B) the bipolar stimulation cuff electrode. The stimulus parameter was set at pulse amplitude $=0.6 \mathrm{~mA}$, pulse width $=0.5 \mathrm{~ms}$, and pulse frequency $=10 \mathrm{~Hz}$. A train $(20 \mathrm{~s})$ of cathode-first, alternating monophasic pulses was delivered to the nerve. The time series represent the averaged response waveform from each of the 3 animals. The yellow dashed box indicates the stimulus artifact. The red arrow indicates the visually identified peak response of VNS-evoked CNAP. When the stimulation cuff electrode was placed rostral to the recording cuff electrode, the amplitude of evoked nerve potential was greater under efferent VNS than afferent VNS. When the stimulation cuff electrode was placed caudal to the recording cuff electrode, the amplitude of evoked nerve potential was greater under afferent VNS than efferent VNS

independently. As illustrated in Figure 1C, each stimulation setting was comprised of a duty cycle of 1-minute ON and 1-minute OFF, and different VNS parameters were performed in a randomized order to eliminate any causal effect of one setting on the other. No stimulus was delivered during the OFF period. As a result, every rat in each group underwent VNS with 24 different sets of parameters.

To examine the effect of high-dose efferent VNS, we further applied VNS ( $1 \mathrm{~mA}, 0.5 \mathrm{~ms}, 10 \mathrm{~Hz}$ ) to 1 rat in the efferent VNS group after which the 24 sets of VNS parameters were delivered. The stimulation was performed with a duty cycle of 30-second ON and 30-second OFF and was repeated for 7 cycles, during which dynamic gastric MRI was continuously performed to assess the effect of stimulation.

\section{6 | CNAP recording and analysis}

To examine the directionality of VNS-evoked CNAP, 3 rats (body weight: $470-510 \mathrm{~g}$ ) underwent the identical surgical procedure as described in the previous section except that two bipolar cuff electrodes were implanted onto the left cervical vagus. The two cuff electrodes were placed $3 \mathrm{~mm}$ apart. In one setting (Figure 2A), the rostral cuff electrode and caudal cuff electrode was used as the stimulation and recording electrode, respectively, to measure evoked CNAP in the efferent pathway. In another setting (Figure $2 \mathrm{~B}$ ), the two electrodes were reversely configured to measure evoked CNAP in the afferent pathway. The stimulation leads were connected to the output of the current stimulator, and the recording leads were connected to the inputs of a differential amplifier (A-M Systems Model 3000; A-M Systems). A data acquisition board (cDAQ-9171; National Instruments) was used to interface with a computer running Matlab R2015a (Mathworks) for recording.

Compound nerve action potential was recorded and averaged using the measured response to a 20-second train of cathode-first, alternating monophasic stimulation (PA $=0.6 \mathrm{~mA}, \mathrm{PW}=0.5 \mathrm{~ms}$, $\mathrm{PF}=10 \mathrm{~Hz}$ ). The measured neural signal was band-pass filtered from $1 \mathrm{~Hz}$ to $20 \mathrm{kHz}$ and then sampled at $10 \mathrm{kHz}$. The neural signal was then processed using an in-house software developed in Matlab. Firstly, the neural signal was notch filtered at $60 \mathrm{~Hz}$ and then segmented into 200 periods of responses (ie, $10 \mathrm{~Hz} \times 20 \mathrm{sec}$ onds $=200$ ). Each period of neural signal was further segmented and clustered into response evoked by the cathodal or anodal pulse, as illustrated in Figure 2. To remove any DC offset, the segmented neural signal was subtracted by the prestimulus baseline, that is, the average amplitude during the prestimulus period ( $5 \mathrm{~ms})$. Then, the signal was averaged across all segments for cathodal and anodal phases, respectively. Finally, the peak amplitude of evoked nerve potential was visually identified (Figure $2 A, B$, indicated by the red arrows). The selectivity of monophasic VNS was computed using the following formula,

$$
\text { Selectivity }=\frac{V_{\text {cathode }}-V_{\text {anode }}}{V_{\text {cathode }}+V_{\text {anode }}}
$$


where $V_{\text {cathode }}$ and $V_{\text {anode }}$ are the amplitude of nerve potential evoked by the cathodal and anodal pulses, respectively.

\section{7 | Image analysis}

The gastric antrum, pylorus, and the proximal duodenum were segmented from the lumen-enhanced MRI images (Figure $3 \mathrm{~A}$ ) by using a custom-built pipeline in Matlab developed in our previous work. ${ }^{15}$ Gastric volumes obtained at different times were rigidly co-registered to the first volume to minimize any motion-induced displacements using Matlab's function imregister. To quantify antral motility, a cross-sectional plane that is perpendicular to the direction of propagating antral contraction waves was defined at $5.625 \mathrm{~mm}$ distant to the pylorus. The segmented voxels within the cross-sectional plane were summed to quantify the cross-sectional area (CSA) of the lumen (Figure 3B). When the antral contraction wave arrived at the cross-sectional plane, a minimal CSA was attained. When the antral contraction moved away from the cross-sectional plane, a maximal CSA was attained. For every rat studied in this study, occlusive contractions often occurred along the lesser curvature, whereas the greater curvature mostly exhibited non-occlusive contractions.

The frequency of antral contraction waves (ACWs) was quantified by measuring the number of contractions occurred at the cross-sectional plane per minute (CPM). The amplitude of ACWs was quantified by computing the mean difference between maximal and minimal CSAs within each 1-minute interval $\left(\Delta \mathrm{CSA} ; \mathrm{mm}^{2}\right)$. The velocity of the ACWs was quantified by calculating the distance that an ACW traveled divided by the duration $\left(\mathrm{mm} \mathrm{s}^{-1}\right)$. On the other hand, pyloric motility was quantified as the area under the curve (AUC) of

\section{(A) Gastric MRI}

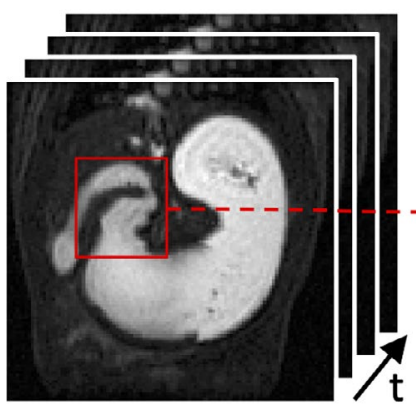

FIGURE 3 Image analysis of gastric motility. (A) Example images obtained from oral contrast-enhanced MRI. (B) Cross-sectional area (CSA) of the antrum ( $5.625 \mathrm{~mm}$ distant from the pylorus) and the luminal CSA of the pylorus were computed from the 3D gastric volume. When an occlusive contraction (as highlighted by the yellow arrow) wave arrived at the cross-sectional plane, the minimal antral CSA was attained. On the other hand, when the occlusive contraction wave was fully away from the cross-sectional plane, the maximal antral CSA was attained. Antral contraction amplitude was defined as the area difference between the maximum and minimum antral CSA. Pyloric motility was defined as the area under the curve of changes in pyloric CSA, which captures both the extent and duration of pyloric opening changes in luminal CSA at the pyloric sphincter. Luminal CSA at the pyloric sphincter was computed for every volume using an approach described in our previous study, ${ }^{15}$ resulting in a contraction time series that represented the changes of luminal CSA over time. An integral analysis was applied to the contraction time series to obtain its AUC. Additionally, the frequency of pyloric opening was quantified by measuring the number of peaks in the contraction time series within each 1-minute interval (contractions per minute; CPM).

\subsection{Data analysis}

For each stimulation group, antral motility (ie, occlusive contraction amplitude) and pyloric motility (ie, AUC) were computed for each stimulus parameter setting during the 1-minute stimulation $\mathrm{ON}$ period. The motility measurements were normalized against their baseline values over the 3-5 minutes immediately preceding the onset of the first VNS setting. The results were expressed as percentage increase/decrease from baseline. The motility measurements during inter-rest periods were also normalized against their baseline values for evaluation of poststimulation effect. Further, a linear regression analysis was performed to characterize the association between stimulus dose and gastric motility. The independent variable used for the regression model was the logarithm-transformed stimulus dose, and the dependent variable was the mean antral contraction amplitude change or mean pyloric motility change. The linear regression analysis was conducted separately for the two stimulation frequencies (ie, $5 \mathrm{~Hz}$ and $10 \mathrm{~Hz}$ ).

\section{9 | Statistics}

All data are expressed as Mean (Standard Error of Mean; SEM). Non-parametric statistical analyses were performed using Matlab Statistics Toolbox due to a small sample size. A non-parametric test (Wilcoxon rank-sum test) was used to compare baseline motility measurements between afferent VNS and efferent VNS groups. For each experiment group, a non-parametric test (Friedman test) was conducted to compare repeated motility measurements during each VNS setting to the same variable obtained during the baseline period, followed by post hoc Wilcoxon sign-rank test. The same nonparametric test was applied to the repeated motility measurements during inter-rest periods to assess any differences from baseline motility measurements. A Student's $t$ test was applied to the slope obtained from regression analysis to test the goodness of fit of the regression model. Pearson correlations between the stimulus dose and the motility measurements were calculated. Values of $P<.05$ were considered statistically significant.

\section{3 | RESULTS}

In this study, we used oral contrast-enhanced MRI to investigate the effect of left cervical VNS on gastric motility in rats. The effect of 
VNS was studied under different stimulus parameters and polarity of electrical stimulation. To counterbalance ongoing gastric physiology during digestion and any potential carryover effects of one VNS trial on the other, the order of 24 VNS settings was randomized within and across animals. Note that 1 rat from the afferent VNS group was excluded due to degraded image quality caused by abnormal respiratory pattern during imaging. Table 1 shows baseline motility measurements. The overall gastric motility was slightly greater in the efferent VNS group than in the afferent VNS group, though the difference was not significant (antral motility (frequency): $P=.14$; antral motility (amplitude): $P=.34$; antral motility (velocity): $P=.64$; pyloric motility (frequency): $P=.45$; pyloric motility (AUC): $P=.99$ ). There were also no significant differences between baseline motility and motility repeatedly measured during inter-rest periods in the afferent VNS group (antral motility (frequency): $P=.34$; antral motility (amplitude): $P=.14$; antral motility (velocity): $P=.24$; pyloric motility (frequency): $P=.75$; pyloric motility (AUC): $P=.37$ ) and in the efferent VNS group (antral motility (frequency): $P=.77$; antral motility (amplitude): $P=.07$; antral motility (velocity): $P=.57$; pyloric motility (frequency): $P=.88$; pyloric motility (AUC): $P=.46$ ). Table 2 shows percentage change of motility from baseline for each VNS setting. We found that left cervical VNS could impose either excitatory or relaxatory effect on gastric motility, depending on both stimulus parameters and the configuration of the placement of the cathode on the bipolar electrode (ie, polarity).

\subsection{Directionality of VNS}

To examine the directionality of VNS-evoked CNAP, we recorded CNAP at the left cervical vagus with the recording cuff electrode placed either rostral or caudal to the stimulation cuff electrode (Figure 2). The cathodal and anodal phases of stimulation artifact have an identical shape but opposite polarity (indicated by the yellow dashed box). When the stimulation cuff electrode was placed rostral to the recording cuff electrode (Figure 2A, left and middle panel), the peak amplitude of VNS-evoked CNAP was greater when the distal lead was connected as the cathode (ie, biased for efferent VNS) than when the proximal lead was connected as the cathode (ie, biased for afferent VNS). When the stimulation cuff electrode was placed caudal to the recording cuff electrode (Figure 2B, left and middle panel), the peak amplitude of VNS-evoked CNAP was greater when the proximal lead was connected as the cathode than when the distal lead was connected as the cathode. The conduction velocity of the evoked potential was about 1-2 m/s, which was likely attributed to activation of unmyelinated $C$ fibers. Figure $2 \mathrm{~A}$ (right panel) and Figure $2 \mathrm{~B}$ (right panel) summarize the peak amplitudes of VNSevoked CNAP under the rostral/caudal electrode configurations and the afferent/efferent VNS conditions. When the stimulation electrode was placed rostral to the recording electrode, the amplitude of measured CNAP evoked by efferent VNS $(0.084 \pm 0.049 \mathrm{mV})$ was greater than that evoked by afferent VNS $(0.017 \pm 0.010 \mathrm{mV})$; the selectivity was about $67 \%$. When the stimulation electrode was placed caudal to the recording electrode, the amplitude of measured CNAP evoked by afferent VNS $(0.105 \pm 0.031 \mathrm{mV})$ was greater than that evoked by efferent VNS $(0.021 \pm 0.021 \mathrm{mV})$; the selectivity was about $82 \%$.

\subsection{VNS effects on antral motility}

For afferent VNS, the 24 sets of parameters primarily induced excitatory effect on antral contraction amplitude to various degree (Figure 4A). At a stimulation frequency of $5 \mathrm{~Hz}$, there was a significant, positive change in contraction amplitude from baseline values under most stimulus settings. A non-significant decrease $(P=.81)$ in contraction amplitude was found when increasing the stimulus dose (Q: product of pulse amplitude and pulse width) at this frequency. However, at the stimulus frequency of $10 \mathrm{~Hz}$, the contraction amplitude continued to fall as the pulse width was increased up to $0.5 \mathrm{~ms}$. Under this circumstance, an increase of VNS dose induced a significant decrease in contraction amplitude ( $t=-3.03, P<.05, R^{2}=.48$ ), but the amplitude was overall still greater than baseline values.

Efferent VNS imposed two different effects on antral motility: one being excitatory and the other being relaxatory, as illustrated in Figure 4B. At both stimulus frequencies, lower doses of VNS promoted contraction amplitude. However, the results from linear regression $\left(t=-7.68, P<.01, R^{2}=.86\right.$ for $5 \mathrm{~Hz} ; t=-4.55, P<.01$, $R^{2}=.67$ for $10 \mathrm{~Hz}$ ) indicated a significant, proportional decrease in contraction amplitude as the stimulus dose increased. A cutoff value $Q$ was defined when the regression line crossed the zero percentage change. The cutoff was similar for both stimulus frequencies

\begin{tabular}{|c|c|c|c|c|c|}
\hline \multirow[b]{2}{*}{ Group } & \multicolumn{3}{|c|}{ Antral motility } & \multicolumn{2}{|c|}{ Pyloric motility } \\
\hline & $\begin{array}{l}\text { Frequency } \\
\text { (CPM) }\end{array}$ & $\begin{array}{l}\text { Amplitude } \\
\left(\triangle \mathrm{CSA} ; \mathrm{mm}^{2}\right)\end{array}$ & $\begin{array}{l}\text { Velocity } \\
\left(\mathrm{mm} \mathrm{s}^{-1}\right)\end{array}$ & $\begin{array}{l}\text { Frequency } \\
\text { (CPM) }\end{array}$ & $\begin{array}{l}\text { AUC } \\
\left(\mathrm{mm}^{2} \mathrm{~s}\right)\end{array}$ \\
\hline $\begin{array}{l}\text { Afferent VNS } \\
(N=7)\end{array}$ & $4.74(0.22)$ & 7.67 (1.28) & $0.62(0.05)$ & $5.17(0.35)$ & $1.98(0.44)$ \\
\hline $\begin{array}{l}\text { Efferent VNS } \\
(\mathrm{N}=5)\end{array}$ & $5.12(0.14)$ & $9.88(1.81)$ & $0.67(0.05)$ & $5.72(0.51)$ & $1.87(0.15)$ \\
\hline
\end{tabular}

TABLE 1 Mean motility measurements in rats under baseline period

Note: Data are presented in mean (standard error of mean; SEM).

Abbreviations: AUC, area under the curve; CPM, contractions per minute; CSA, cross-sectional area. 
$(\mathrm{Q}=0.0429 \mu \mathrm{C}$ for $5 \mathrm{~Hz}$ and $\mathrm{Q}=0.0435 \mu \mathrm{C}$ for $10 \mathrm{~Hz})$, suggesting that a stimulus intensity greater than respective values would cause relaxatory effect on antral motility.

Interestingly, when afferent VNS and efferent VNS were performed alternately, the stimulus-response relations show an additive effect of afferent and efferent VNS, with the effect of efferent VNS being more dominant over afferent VNS (Figure 4C). Regression analysis revealed a significant decrease in contraction amplitude as the stimulus dose increased $\left(t=-3.55, P<.01, R^{2}=.56\right.$ for $5 \mathrm{~Hz}$; $t=-2.82, P<.05, R^{2}=.46$ for $\left.10 \mathrm{~Hz}\right)$. Here, a slightly higher cutoff $\mathrm{Q}$

TAB LE 2 Mean percentage change in motility for each set of VNS parameters for the 3 conditions

\begin{tabular}{|c|c|c|c|c|c|c|}
\hline \multirow[b]{3}{*}{ Group } & \multirow{3}{*}{$\begin{array}{l}\mathrm{PA} \\
(\mathrm{mA})\end{array}$} & \multirow{3}{*}{$\begin{array}{l}\text { PW } \\
\text { (ms) }\end{array}$} & \multirow{2}{*}{\multicolumn{2}{|c|}{$\frac{\% \text { Change in antral contraction amplitude }}{\text { PF }(\mathrm{Hz})}$}} & \multicolumn{2}{|c|}{$\%$ Change in pyloric motility (AUC) } \\
\hline & & & & & \multicolumn{2}{|l|}{$\mathrm{PF}(\mathrm{Hz})$} \\
\hline & & & 5 & 10 & 5 & 10 \\
\hline \multirow{12}{*}{$\begin{array}{l}\text { Afferent VNS } \\
(\mathrm{N}=7)\end{array}$} & \multirow[t]{3}{*}{0.13} & 0.13 & $31.02(13.81)$ & $72.79(8.72)^{*}$ & $45.43(42.10)$ & $39.86(52.17)$ \\
\hline & & 0.25 & $50.94(38.63)$ & $47.35(6.04)^{*}$ & $20.22(18.06)$ & $25.08(35.64)$ \\
\hline & & 0.5 & $69.89(12.17)^{*}$ & $25.58(17.02)$ & $29.26(40.14)$ & $24.35(24.51)$ \\
\hline & \multirow[t]{3}{*}{0.25} & 0.13 & $21.96(13.52)$ & $44.47(13.79)^{*}$ & $38.40(32.44)$ & $5.41(29.75)$ \\
\hline & & 0.25 & $46.46(13.58)^{*}$ & $46.92(6.29)^{*}$ & $40.92(54.80)$ & $47.93(33.70)$ \\
\hline & & 0.5 & $53.18(19.93)^{*}$ & $57.25(17.14)$ & $107.24(50.92)$ & $76.36(64.46)$ \\
\hline & \multirow[t]{3}{*}{0.5} & 0.13 & 42.19 (11.78) & $74.62(22.26)^{*}$ & $-4.37(30.32)$ & $20.26(30.59)$ \\
\hline & & 0.25 & 51.45 (20.39) & 50.53 (17.28) & 42.85 (42.95) & $54.50(43.52)$ \\
\hline & & 0.5 & $37.10(13.49)^{*}$ & $2.48(8.58)$ & $54.32(54.64)$ & $142.71(60.96)$ \\
\hline & \multirow[t]{3}{*}{1} & 0.13 & $45.08(10.78)^{*}$ & $52.93(26.82)$ & $114.60(79.14)$ & $75.34(60.17)$ \\
\hline & & 0.25 & $43.37(22.47)$ & $24.86(25.51)$ & $53.83(32.52)$ & $94.72(19.47)^{*}$ \\
\hline & & 0.5 & $21.62(13.46)$ & $-5.78(14.72)$ & 171.33 (105.48) & $58.64(60.54)$ \\
\hline \multirow{12}{*}{$\begin{array}{l}\text { Efferent VNS } \\
(\mathrm{N}=5)\end{array}$} & \multirow[t]{3}{*}{0.13} & 0.13 & $22.99(9.90)$ & $29.46(18.98)$ & $20.00(30.88)$ & 1.91 (39.89) \\
\hline & & 0.25 & $15.43(20.00)$ & $27.03(28.36)$ & $62.10(30.19)$ & $68.10(53.95)$ \\
\hline & & 0.5 & $-26.91(13.71)$ & $-5.10(20.07)$ & $136.43(109.46)$ & $75.26(28.51)$ \\
\hline & \multirow[t]{3}{*}{0.25} & 0.13 & $5.48(12.23)$ & $-14.94(13.40)$ & $57.46(29.01)$ & $132.55(56.64)$ \\
\hline & & 0.25 & -10.02 (15.99) & $-26.85(17.66)$ & 129.02 (48.11) & $63.20(71.85)$ \\
\hline & & 0.5 & $-17.95(20.88)$ & $0.60(26.11)$ & $86.55(55.50)$ & 114.57 (79.73) \\
\hline & \multirow[t]{3}{*}{0.5} & 0.13 & $-4.60(14.82)$ & $-16.60(11.88)$ & $60.73(43.41)$ & $198.72(39.70)^{*}$ \\
\hline & & 0.25 & $-35.20(4.69)^{*}$ & $-22.49(20.35)$ & $50.04(39.27)$ & 107.41 (69.88) \\
\hline & & 0.5 & $-41.20(13.82)$ & $-35.74(9.22)^{*}$ & $190.76(54.63)^{*}$ & $155.06(70.12)$ \\
\hline & \multirow[t]{3}{*}{1} & 0.13 & $-32.73(11.27)$ & $-34.77(17.41)$ & $200.06(85.45)^{*}$ & $195.98(53.79)^{*}$ \\
\hline & & 0.25 & $-26.78(11.34)$ & $-46.74(20.54)$ & $155.71(78.32)$ & $157.24(58.10)$ \\
\hline & & 0.5 & $-54.92(11.35)^{*}$ & $-55.00(10.63)^{*}$ & $169.53(75.85)$ & $196.94(102.67)$ \\
\hline \multirow{12}{*}{$\begin{array}{l}\text { Combined afferent } \\
\text { and efferent VNS } \\
(\mathrm{N}=7)\end{array}$} & \multirow[t]{3}{*}{0.13} & 0.13 & $3.27(9.37)$ & $0.46(19.10)$ & $4.68(26.34)$ & $99.29(28.37)^{*}$ \\
\hline & & 0.25 & $33.33(30.54)$ & $21.16(17.81)$ & $38.46(32.26)$ & $81.17(65.84)$ \\
\hline & & 0.5 & $-9.64(8.75)$ & $22.32(25.96)$ & $42.17(41.57)$ & $44.15(33.02)$ \\
\hline & \multirow[t]{3}{*}{0.25} & 0.13 & $-0.35(14.94)$ & $-18.76(12.65)$ & $155.68(58.57)$ & $26.73(28.78)$ \\
\hline & & 0.25 & $5.41(12.98)$ & $-10.90(14.43)$ & $152.46(102.21)$ & $94.05(86.24)$ \\
\hline & & 0.5 & $-11.37(14.46)$ & $-7.60(18.56)$ & $224.57(79.41)^{*}$ & $124.25(85.36)$ \\
\hline & \multirow[t]{3}{*}{0.5} & 0.13 & $-0.19(12.18)$ & $-11.45(11.21)$ & 111.07 (88.34) & 115.32 (78.57) \\
\hline & & 0.25 & $-22.30(9.51)^{*}$ & $-17.94(14.70)$ & $189.58(95.54)$ & 171.04 (53.73) \\
\hline & & 0.5 & $-11.61(17.66)$ & $-35.23(13.79)$ & $266.36(86.14)^{*}$ & $232.57(100.82)$ \\
\hline & \multirow[t]{3}{*}{1} & 0.13 & $-24.70(10.18)^{*}$ & $-21.50(8.74)^{*}$ & $137.90(61.66)$ & 113.29 (75.09) \\
\hline & & 0.25 & $-37.90(10.92)^{*}$ & $-23.03(13.66)$ & $182.80(56.07)^{*}$ & $175.93(72.64)$ \\
\hline & & 0.5 & $-30.31(7.23)^{*}$ & $-34.41(11.49)^{*}$ & 205.21 (86.33) & 161.72 (61.61) \\
\hline
\end{tabular}

Note: Data are presented in mean (standard error of mean; SEM). An asterisk indicates a significant value $(P<.05)$.

Abbreviations: AUC, area under the curve; PA, pulse amplitude; PF, pulse frequency; PW, pulse width. 


\section{(A) Afferent VNS}

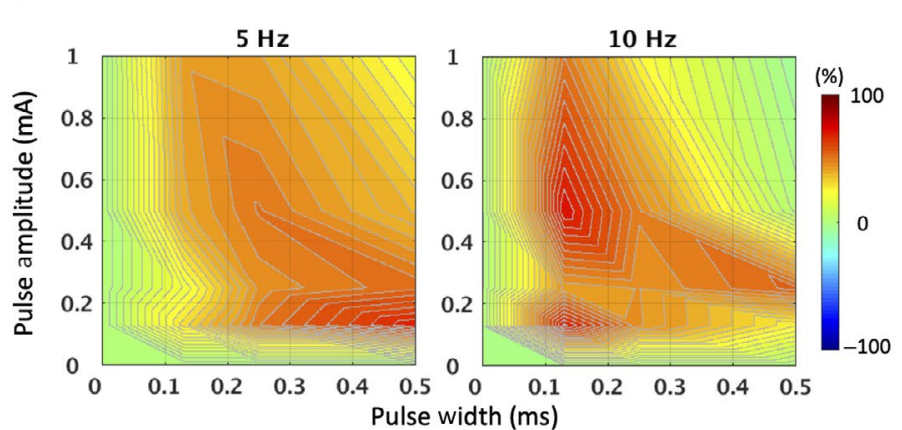

(B) Efferent VNS

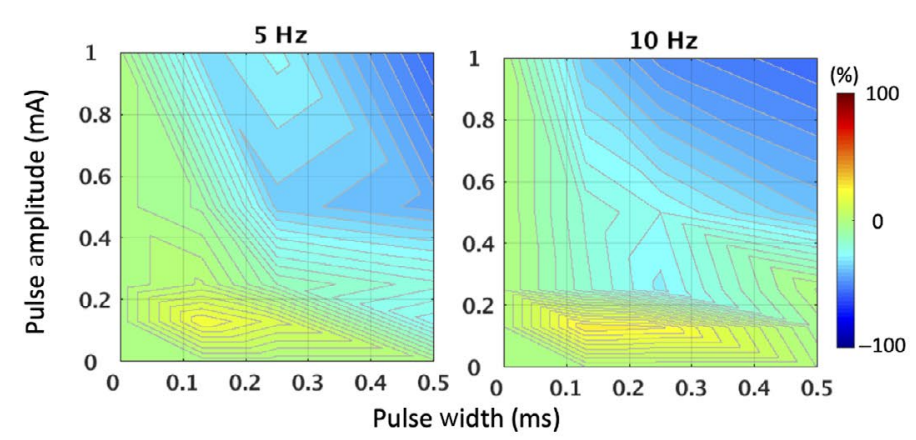

\section{(C) Combined afferent \& efferent VNS}
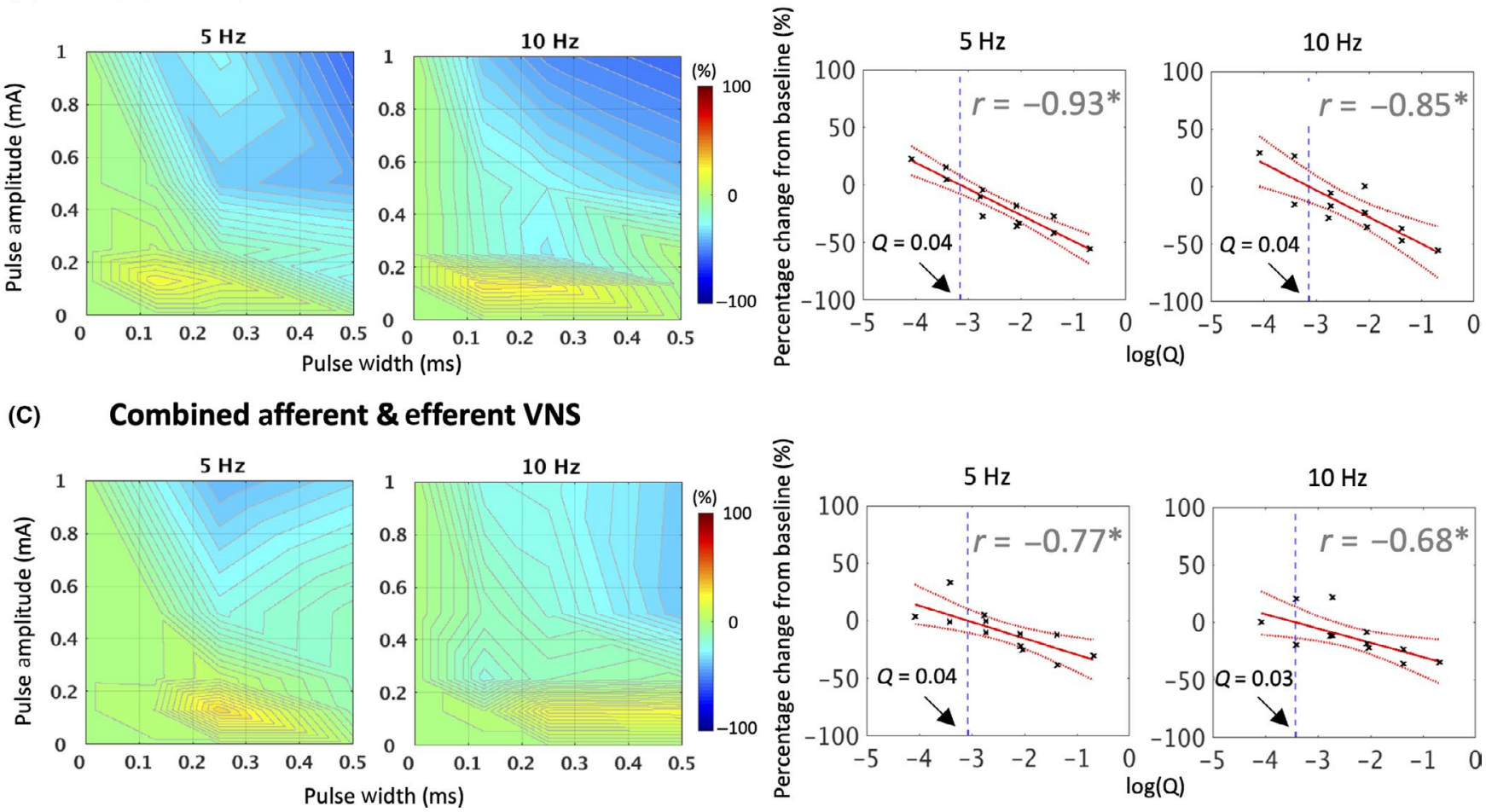

FIGURE 4 Effects of VNS parameters and polarity of VNS on antral motility. (A) Afferent VNS. (B) Efferent VNS. (C) Combined Afferent and Efferent VNS. Left panel: Effect of VNS on antral contraction amplitude under different VNS parameters. Right panel: Linear regression analysis of changes in antral contraction amplitude as a function of log-transformed VNS dose (Q: pulse amplitude $\times$ pulse width). Afferent VNS promoted antral contraction amplitude more effectively than efferent VNS. Meanwhile, high-dose efferent VNS inhibited antral contraction. For both efferent and combined afferent and efferent VNS, a cutoff value Q (dotted blue line) was defined when the regression line crossed the zero percentage change. The color bar indicates percentage change of antral contraction amplitude from baseline values. Dotted red lines: $95 \%$ confidence interval of regression. $r$ : Pearson correlation coefficient. ${ }^{*} P<.05$

was found for the stimulus frequency at $5 \mathrm{~Hz}(\mathrm{Q}=0.0461 \mu \mathrm{C})$ than at $10 \mathrm{~Hz}(\mathrm{Q}=0.0328 \mu \mathrm{C})$, suggesting that the threshold for transitioning from excitatory to relaxatory effect was lower at $10 \mathrm{~Hz}$ than at $5 \mathrm{~Hz}$.

\section{3 | Effects of strong VNS dose on gastric physiology}

As revealed in Figure 4, we found that high-dose efferent (and combined afferent and efferent VNS) dampened antral contraction amplitude. To further demonstrate the effect, we additionally performed high-dose efferent VNS (1 mA, $0.5 \mathrm{~ms}$, $10 \mathrm{~Hz}$ ) with 30-second ON, 30-second OFF duty cycle in 1 animal in the efferent VNS group. As illustrated in Figure 5A, when efferent VNS at supramaximal intensity was applied to the left cervical vagus nerve, an enlarged corporal and antral volume was induced. Furthermore, antral contraction waves ceased during the stimulation ON period. Figure 5B shows an example CSA change extracted from antrum before, during and after efferent VNS. At the onset of each VNS, an abrupt change in CSA can be observed, reflecting the enlargement of antral volume caused by relaxation 
(A)

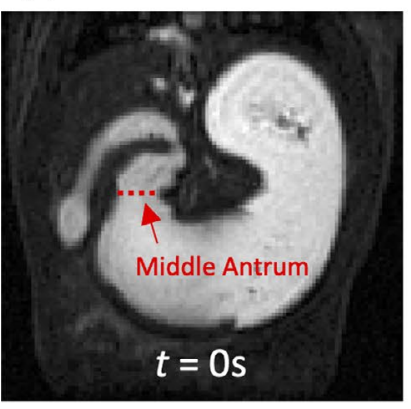

Efferent VNS

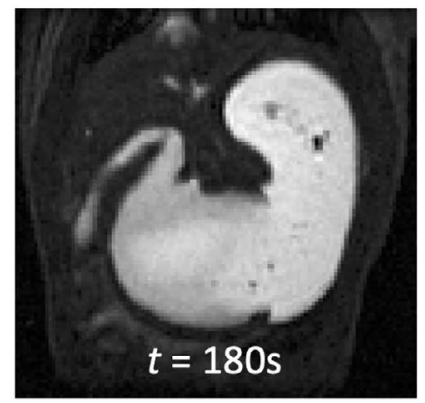

(B)

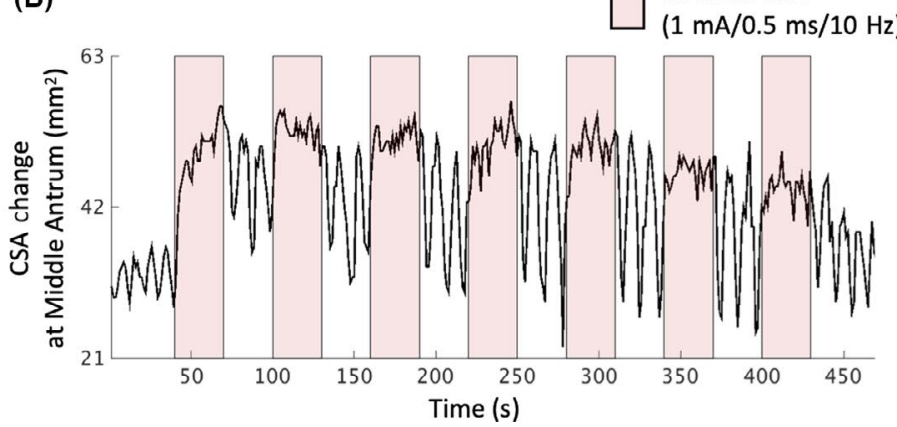

FIGURE 5 High-dose efferent VNS retards antral peristalsis. (A) Example gastric images before and during high-dose efferent VNS (pulse amplitude $=1 \mathrm{~mA}$, pulse width $=0.5 \mathrm{~ms}$, pulse frequency $=10 \mathrm{~Hz}$ ). Efferent VNS under this parameter setting ceased antral contraction wave while enlarging corporal and antral volume likely through relaxation. A dynamic illustration of this process can be found in Video S1. (B) A CSA change taken from the middle antrum as indicated in panel (A). The onset of high-dose efferent VNS significantly increased the CSA, followed by little or no phasic change in CSA. There was a large rebound, intensive contraction on the offset of efferent VNS

of the antrum. The propagation of antral contraction waves ceased during efferent VNS. Such inhibitory effect on antral contraction was immediately followed by intensive, rebound contractions when the VNS was turned off. The effect was reproducible over multiple cycles of stimulation. Video S1 demonstrates the dynamic change in antral contraction waves during high-dose efferent VNS.

\subsection{VNS effects on pyloric motility}

Regardless of the polarity of VNS, the vagal innervation on pyloric sphincter was found to be mostly relaxatory (corresponding to a decrease in pyloric tone, or equivalently to an increase in AUC). For afferent VNS at $5 \mathrm{~Hz}$, the maximum response was induced either at the highest PA (1 mA) or the highest PW $(0.5 \mathrm{~ms})$ utilized in this study. When stimulating at $10 \mathrm{~Hz}$, the greatest response was observed at $\mathrm{PA}=0.5 \mathrm{~mA}$ and $\mathrm{PW}=0.5 \mathrm{~ms}$, and the effective $\mathrm{PA}$ and $\mathrm{PW}$ for promoting pyloric motility were of wider range than that when stimulating at $5 \mathrm{~Hz}$. An increase in stimulus dose induced a significant increase in pyloric AUC at both stimulus frequencies $(t=2.35$, $P<.05, R^{2}=.36$ for $5 \mathrm{~Hz} ; t=2.78, P<.05, R^{2}=.50$ for $10 \mathrm{~Hz}$ ), as shown in Figure 6A. On the other hand, efferent VNS was found to promote pyloric opening more effectively under a wider spectrum of VNS settings than afferent VNS (Figure 6B). An increase in stimulus dose induced a significant increase in pyloric $A \cup C$ at both stimulus frequencies $\left(t=3.32, P<.01, R^{2}=.52\right.$ for $5 \mathrm{~Hz} ; t=3.12, P<.05$, $R^{2}=.50$ for $10 \mathrm{~Hz}$ ).

A marked increase in pyloric motility was found when performing combined afferent and efferent VNS at high dose and at $5 \mathrm{~Hz}$ (Figure 6C). The stimulus-response relations again show additive effect of afferent and efferent VNS. A stimulus frequency of $5 \mathrm{~Hz}$ was found to promote pyloric motility more effectively than $10 \mathrm{~Hz}$. Finally, a significant linear regression indicated a proportional increase in pyloric motility as the stimulus dose increased $\left(t=3.51, P<.01, R^{2}=.55\right.$ for $5 \mathrm{~Hz} ; t=3.20, P<.01$, $R^{2}=.51$ for $10 \mathrm{~Hz}$ ).

\section{4 | DISCUSSION}

In this study, we characterized the effect of different cervical VNS parameters on gastric motility in anesthetized rats. To our knowledge, this is the first animal study that utilizes gastric MRI to non-invasively assess postprandial gastric motility under VNS with a wide range of parameters. Gastric MRI was respiratory-gated such that motion artifacts were minimized ( 1 rat data were excluded due to degraded image quality caused by instable respiration during the experiment). The impact of polarity of VNS was studied by configuring the placement of cathode on the bipolar cuff electrode. Gastric MRI revealed that electrical activation biased for the afferent pathway may promote the amplitude of antral contractions more effectively than biased for the efferent pathway. A reduction in antral contraction amplitude and relaxation of pyloric sphincter under biased efferent VNS highlighted the inhibitory pathway of the motor limb of the vagovagal circuitry.

\subsection{Efferent VNS induces multiple effects on gastric physiology}

Previous studies on vagal control of gastric motility have largely focused on the efferent parasympathetic innervation of the GI tract. $^{10,12,17,18}$ The modulatory role of efferent VNS with different stimulus parameters on gastric motility was discovered by Veach ${ }^{19}$ over a hundred years ago. In a cat model, he found that the stronger the stimulus strength and/or the higher the stimulus frequency, the more prominent the inhibitory effect on gastric motility. The present finding supports the general opinion that there are two groups of efferent fibers that influence gastric motility: one being excitatory and the other being inhibitory. ${ }^{2}$ In line with previous studies, ${ }^{10,20}$ the activation threshold for the two groups of fibers seems to be different, which was dependent on the stimulus strength (pulse amplitude and pulse width). Here, we found that lower pulse amplitude and/or lower pulse width produced excitatory response on antral contraction amplitude. However, when 
(A)

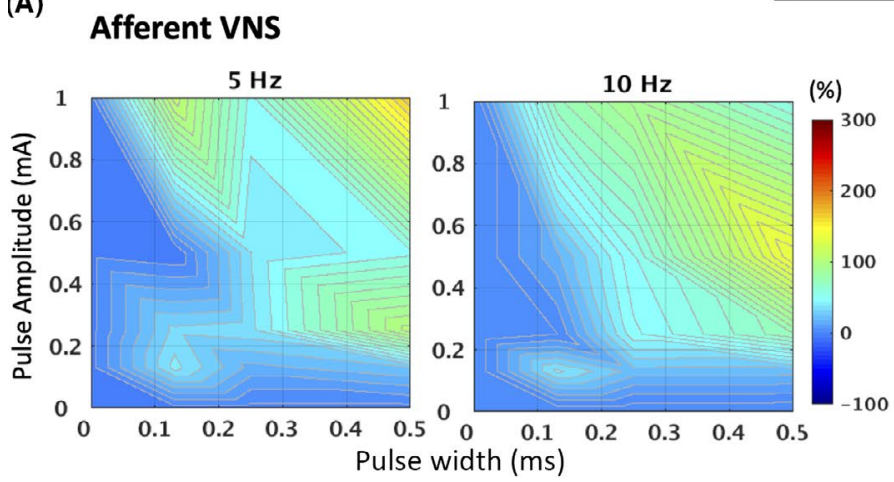

\section{(B) Efferent VNS}

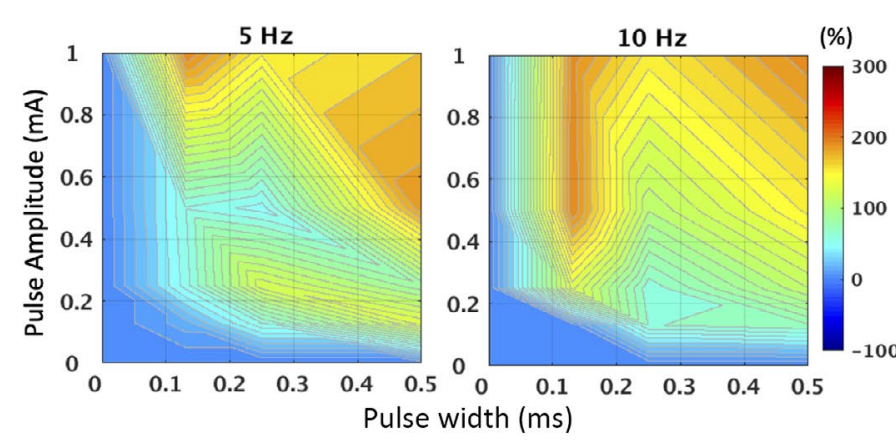

(C)

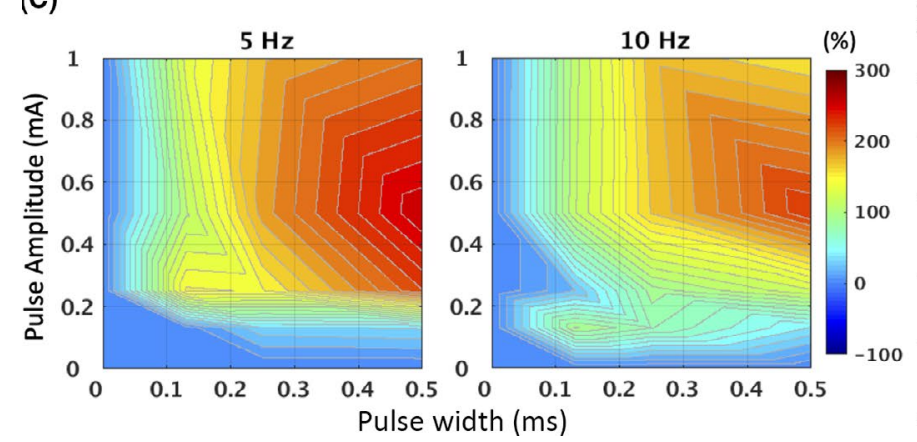

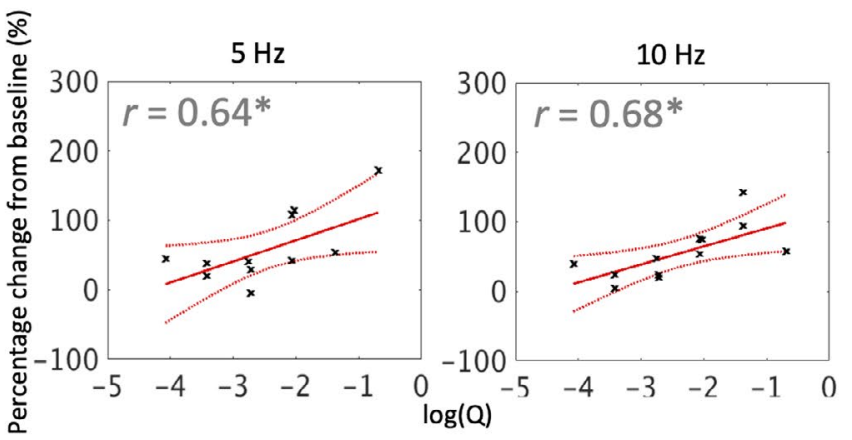

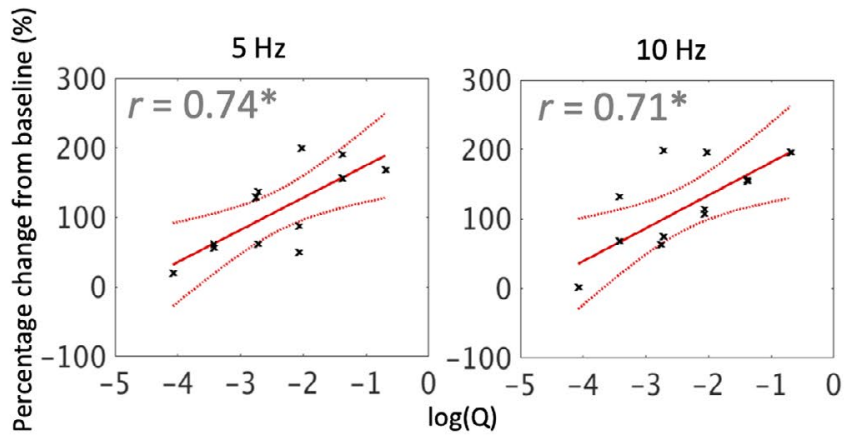

FIGURE 6 Effects of VNS parameters and polarity of VNS on pyloric motility. (A) Afferent VNS. (B) Efferent VNS. (C) Combined Afferent and Efferent VNS. Left panel: Effect of VNS on pyloric motility under different VNS parameters. Right panel: Linear regression analysis of changes in pyloric motility as a function of log-transformed VNS dose (Q: pulse amplitude $\times$ pulse width). An increase in stimulus dose generally promoted pyloric motility, indicating an increase in AUC of pyloric opening (or equivalently a decrease in pyloric tone). The color bar indicates percentage change of AUC of pyloric opening from baseline values. Dotted red lines: $95 \%$ confidence interval of regression. $r$ : Pearson correlation coefficient. ${ }^{*} P<.05$

we continued to increase the pulse amplitude and pulse width over a certain level, the amplitude of antral contraction waves became smaller, and eventually, the contraction waves ceased at supramaximal intensity (Video S1). The phenomenon confirms the presence of efferent fibers with two different calibers; the fiber caliber for inducing excitatory response is perhaps larger than the fiber caliber for inducing inhibitory response, where the activation threshold is lower for the former than the latter. The two fiber groups are likely unmyelinated fibers, as the activation thresholds for the two groups were suggested to be both higher than that needed for inducing cardiac response, ${ }^{21,22}$ under which the heart is primarily mediated by efferent $B$ fibers. Our experimental observation is also consistent with the finding that there are only a few myelinated fibers in the abdominal vagi. ${ }^{23}$ Notably, the pulse amplitude and pulse width required for inducing inhibitory response are generally smaller than previously reported parameters, though different animal species were used. ${ }^{10,12}$ Speculatively, the difference in experimental condition (ie, preprandial versus postprandial state) may be the underlying cause, as the basal gastric tone should vary across the two states. It was also suggested that the stimulus frequency could be responsible for selective activation of the efferent excitatory (cholinergic) and inhibitory (non-adrenergic, non-cholinergic [NANC]) effects ${ }^{24}$ (ie, $5 \mathrm{~Hz}$ for acetylcholine release and $10 \mathrm{~Hz}$ for vasoactive intestinal peptide 
release). Nevertheless, a clear-cut effect on frequency specificity was not apparent in the present study, as the 2 frequencies both produced excitatory and inhibitory response.

The inhibitory motility pattern induced by high-dose efferent VNS consists of two phases. The first phase was a rapid relaxation of the antrum at the onset of stimulus, followed by intensive, rebound contractions on cessation of the stimulation. As reported and discussed elsewhere, ${ }^{12,25}$ the rebound contractions could be of purinergic origin and the release of prostaglandins by NANC terminals may account for this poststimulus pattern.

The influence of efferent VNS on pyloric motility appeared to be primarily inhibitory. This observation coincides with previous findings that VNS does not seem to impose a strong tonic influence on pyloric resistance; VNS was shown to decrease pyloric resistance through the NANC pathways and thus increase transpyloric flow. ${ }^{26}$ However, the seemingly pure inhibitory effect could depend on the choice of stimulus frequency, as it was suggested in a dog study that low-frequency stimulation $(0.2-0.5 \mathrm{~Hz})$ exerted tonic contractions, whereas higher frequency $(>0.7 \mathrm{~Hz})$ stimulation could inhibit both phasic and tonic contractions. ${ }^{27}$ Further experiments are needed to evaluate the potentially excitatory effect of efferent VNS on pyloric tone with a wider range of stimulus frequency.

In sum, our results suggest that electrical stimulation biased for the efferent pathway could induce multiple effects on gastric physiology. These effects were heavily, if not entirely, dependent on the dose of the stimulation. The likelihood that both excitatory and inhibitory efferent fibers are unmyelinated fibers pose technical difficulty in selective activation of one but not the other fiber group. As a result, efferent VNS employs complex and heterogeneous neural signaling to the gut, which may lead to undesired, mixed gastric responses. Furthermore, a promotion of antropyloric coordination seems difficult to achieve, because we found no single parameter setting that can optimally increase antral motility while reducing pyloric tone.

\section{2 | Reflex excitation on gastric motility via afferent VNS}

While most existing studies utilized electrical stimulation of the peripheral end of the sectioned vagal nerve, the effect of afferent VNS on gastric motility has received considerably less attention. Here, we performed VNS at the cervical level that was biased for the afferent pathway to mimic the vagovagal reflex occurring in the physiological state. The vagal afferents carry signals from stretch receptors ${ }^{28,29}$ and chemoreceptors ${ }^{30}$ to dorsal vagal complex in the brainstem, resulting in downstream, coordinated signals to different segments of the GI tract. ${ }^{2,31}$ It has been widely demonstrated that fundic distention could induce reflex excitation on antral motility ${ }^{12,32}$ and pyloric relaxation. ${ }^{33}$ On the other hand, balloon distension of the duodenum could inhibit antral motility and increase pyloric tone. ${ }^{34,35}$ Speculatively, activation of different branches of the vagal afferents may result in selective and choreographic gastric motility. Indeed, we found that afferent VNS at $5 \mathrm{~Hz}$ significantly promoted antral motility under a wide range of stimulus settings. This finding suggests the afferent fibers that are responsible for delivering excitatory response to the antrum may be recruited under this stimulus frequency. When the stimulus frequency was increased to $10 \mathrm{~Hz}$, a higher stimulus dose resulted in a decrease in contraction amplitude, suggesting that a second group of fibers has been recruited. The second vagal afferents may convey reflex signals that return to the stomach to activate relaxation of the smooth muscle via inhibitory vagal efferents, as a similar finding was reported previously. ${ }^{36,37}$ Such a relaxatory response is also consistent with our finding that a decrease in pyloric tone could be achieved at a similar stimulus dose. Taken together, electrical stimulation biased for the vagal afferent pathway into the central nervous system could potentially result in a more coordinated, physiological downstream signaling to different segments of the GI tract than direct efferent VNS.

\section{3 | Limitations and future directions}

There are several limitations in this study. Firstly, all animals were sedated with a combination of dexmedetomidine and low-dose Isoflurane $(<0.5 \%)$. The anesthetic protocol was adopted from a rat brain imaging study that showed better preservation of functional activities in the brain compared to using isoflurane alone. ${ }^{38}$ However, the use of anesthetics could still inevitably have impacted the excitability of the nerve and thus might have modulated gastric motility. Dexmedetomidine, also known as an agonist of $\alpha 2$-adrenergic receptors, has an inhibitory effect on the sympathetic system. ${ }^{39}$ While the sympathetic nervous system primarily plays an inhibitory role on gastric motility, ${ }^{1}$ reducing sympathetic innervations on the $\mathrm{Gl}$ tract may potentially alter the pattern of VNS-induced gastric responses. On the other hand, the use of isoflurane has been shown to significantly reduced gut motility ${ }^{40}$ and transits. ${ }^{41}$ Further experiments may be of interest to evaluate VNS effects on gut motility in animals under alternative anesthetics.

Secondly, although the stimulus-response maps show differential patterns between conditions within (eg, afferent vs combined afferent and efferent VNS) and across (eg, afferent vs efferent VNS) animals, results from electrophysiological recordings indicate that the stimulation was not fully unidirectional. Our results (ie, the amplitude of CNAP evoked by cathodal and anodal pulses was different) suggest that the direction of VNS-evoked CNAP could be biased toward the side where the cathode is located, but there could be sufficient current spread that may lead to bidirectional stimulation, which negates the effect of anodal block. Hence, the interpretation of the effects of different polarity of VNS on gastric motility should be cautiously exercised. Additional experimentation (eg, vagotomy and chemical nerve blockade) is required to better affirm the directionality of VNS.

Last but not least, a majority of VNS-gastric studies were conducted on fasted animals. The pattern of preprandial gastric physiology is relatively more stable and predictable. However, postprandial 
gastric motility is a dynamic process that includes complex feedforward, feedback and local reflexes during digestion of a meal. Although the sequence of 24 VNS settings was randomized within and across animals to counterbalance the impact of spontaneous gastric physiology, it is possible that the VNS-induced gastric response observed in the present study might be confounded by (a) spontaneous changes from the gastric emptying process, and (b) carryover effects from each stimulation trial, especially at high-dose VNS. Although it remains an experimental challenge to screen the effect of various VNS parameters on the same animal within an imaging session, the results presented in this study are expected to guide future VNS studies in rats.

\section{ACKNOWLEDGMENT}

This work was supported in part by NIH SPARC 1OT2TR001965 and

Purdue University.

\section{DISCLOSURE}

All authors report no conflict of interest.

\section{AUTHOR CONTRIBUTIONS}

K. Lu, TL Powley, and Z. Liu conceived the original study. K. Lu and J. Cao collected the data. J. Cao and R. Phillips helped with the surgical procedures. K. Lu analyzed the data. K. Lu prepared the original draft of the manuscript. TL Powley and Z. Liu reviewed and edited the manuscript. All authors contributed to data interpretation, discussion, and comments on the manuscript.

\section{ORCID}

Kun-Han Lu iD https://orcid.org/0000-0002-0355-8515

Terry L. Powley (D) https://orcid.org/0000-0001-6689-7058

Zhongming Liu (iD https://orcid.org/0000-0002-8773-4204

\section{REFERENCES}

1. Browning KN, Travagli RA. Central nervous system control of gastrointestinal motility and secretion and modulation of gastrointestinal functions. Compr Physiol. 2014;4(4):1339-1368.

2. Travagli RA, Anselmi L. Vagal neurocircuitry and its influence on gastric motility. Nat Rev Gastroenterol Hepatol. 2016;13(7):389-401.

3. Berthoud HR, Carlson NR, Powley TL. Topography of efferent vagal innervation of the rat gastrointestinal tract. Am J Physiol Integr Comp Physiol. 1991;260(1):R200-R207.

4. Powley TL, Phillips RJ. Morphology and topography of vagal afferents innervating the Gl tract. Am J Physiol Liver Physiol. 2002;283(6):G1217-G1225.

5. Horn CC, Ardell JL, Fisher LE. Electroceutical targeting of the autonomic nervous system. Physiology. 2019;34(2):150-162.

6. Stakenborg N, Wolthuis AM, Gomez-Pinilla PJ, et al. Abdominal vagus nerve stimulation as a new therapeutic approach to prevent postoperative ileus. Neurogastroenterol Motil. 2017;29(9):1-11.

7. Frøkjær JB, Bergmann S, Brock C, et al. Modulation of vagal tone enhances gastroduodenal motility and reduces somatic pain sensitivity. Neurogastroenterol Motil. 2016;28(4):592-598.

8. Prechtl JC, Powley TL. The fiber composition of the abdominal vagus of the rat. Anat Embryol (Berl). 1990;181(2):101-115.
9. Berthoud HR, Powley TL. Vagal afferent innervation of the rat fundic stomach: morphological characterization of the gastric tension receptor. J Comp Neurol. 1992;319(2):261-276.

10. Martinson J. The effect of graded stimulation of efferent vagal nerve fibres on gastric motility. Acta Physiol Scand. 1964;62(3):256-262.

11. Jansson G, Martinson J. Some quantitative considerations on vagally induced relaxation of the gastric smooth muscle in the cat. Acta Physiol Scand. 1965;63(3):351-357.

12. Andrews PL, Scratcherd T. The gastric motility patterns induced by direct and reflex excitation of the vagus nerves in the anaesthetized ferret. J Physiol. 1980;302(1):363-378.

13. Berthoud HR, Hennig G, Campbell M, Volaufova J, Costa M. Videobased spatio-temporal maps for analysis of gastric motility in vitro: effects of vagal stimulation in guinea-pigs. Neurogastroenterol Motil. 2002;14(6):677-688.

14. Brindley GS, Craggs MD. A technique for anodally blocking large nerve fibres through chronically implanted electrodes. J Neurol Neurosurg Psychiatry. 1980;43(12):1083-1090.

15. Lu K-H, Cao J, Oleson S, Powley TL, Liu Z. Contrast enhanced magnetic resonance imaging of gastric emptying and motility in rats. IEEE Trans Biomed Eng. 2017;64(11):1-1.

16. Lu K-H, Cao J, Oleson S, et al. Vagus nerve stimulation promotes gastric emptying by increasing pyloric opening measured with magnetic resonance imaging. Neurogastroenterol Motil. 2018;30(10): $\mathrm{e} 13380$

17. Grundy D, Scratcherd T. Effect of stimulation of the vagus nerve in bursts on gastric acid secretion and motility in the anaesthetized ferret. J Physiol. 1982;333:451-461.

18. Takahashi T, Owyang C. Vagal control of nitric oxide and vasoactive intestinal polypeptide release in the regulation of gastric relaxation in rat. J Physiol. 1995;484(2):481-492.

19. Veach HO. Studies on the innervation of smooth muscle. I. Vagus effects on the lower end of the esophagus, cardia and stomach of the cat, and the stomach and lung of the turtle in relation to Wedensky inhibition. Am J Physiol. 1925;71(2):229-264.

20. Aihara Y, Nakamura H, Sato A, Relations AS. Between various fiber groups of vagal and spanchnic nerves and gastric motility in rats. Neurosci Lett. 1978;10:281-286.

21. Martinson J, Muren A. Excitatory and inhibitory effects of vagus stimulation on gastric motility in the cat. Acta Physiol Scand. 1963;57(4):309-316.

22. Wasilczuk KM, Qing KY, Irazoqui PP, et al. B fibers are the best predictors of cardiac activity during Vagus nerve stimulation. Bioelectron Med. 2018;4(1):1-11.

23. Gabella G, Pease HL. Number of axons in the abdominal vagus of the rat. Brain Res. 1973;58(2):465-469.

24. Bockx I, Verdrengh K, Vander Elst I, et al. High-frequency vagus nerve stimulation improves portal hypertension in cirrhotic rats. Gut. 2012;61(4):604-612.

25. Baccari MC, Calamai F, Staderini G. The influence of the vagally induced rebound contractions on the non-adrenergic, non-cholinergic (NANC) inhibitory motility of the rabbit stomach and the role of prostaglandins. J Auton Nerv Syst. 1992;37(2):125-135.

26. Malbert $\mathrm{CH}$, Mathis $\mathrm{C}$, Laplace JP. Vagal control of pyloric resistance. Am J Physiol Liver Physiol. 1995;269(4):G558-G569.

27. Allescher H-D, Daniel EE, Dent J, Fox JET, Kostolanska F. Extrinsic and intrinsic neural control of pyloric. J Physiol. 1988;401:17-38.

28. Powley TL, Hudson CN, Mcadams JL, Baronowsky EA, Phillips RJ. Vagal intramuscular arrays: the specialized mechanoreceptor arbors that innervate the smooth muscle layers of the stomach examined in the rat. J Comp Neurol. 2016;524(4):713-737.

29. Powley TL, Baronowsky EA, Gilbert JM, et al. Vagal afferent innervation of the lower esophageal sphincter. Auton Neurosci Basic Clin. 2013;177(2):129-142. 
30. Berthoud HR, Neuhuber WL. Functional and chemical anatomy of the afferent vagal system. Auton Neurosci Basic Clin. 2000;85(1-3):1-17.

31. Travagli RA, Hermann GE, Browning $\mathrm{KN}$, Rogers RC. Brainstem circuits regulating gastric function. Annu Rev Physiol. 2006;68(1):279-305.

32. Andrews PLR, Grundy D, Scratcherd T. Reflex excitation of antral motility induced by gastric distension in the ferret. J Physiol. 1980;298:79-84.

33. Ishiguchi T, Nakajima M, Sone H, Tada H, Kumagai AK, Takahashi T. Gastric distension-induced pyloric relaxation: central nervous system regulation and effects of acute hyperglycaemia in the rat. $J$ Physiol. 2004;533(3):801-813.

34. Shafik A. Effect of duodenal distension on the pyloric sphincter and antrum and the gastric corpus: duodenopyloric reflex. World J Surg. 1998;22(10):1061-1064.

35. Treacy PJ, Jamieson GG, Dent J. The effect of duodenal distension upon antro-pyloric motility and liquid gastric emptying in pigs. ANZ J Surg. 2008;66(1):37-40.

36. Deloof S, Bennis M, Rousseau JP. Inhibition of antral and pyloric electrical activity by vagal afferent stimulation in the rabbit. J Auton Nerv Syst. 1987;19(1):13-20.

37. Deloof S, Croix D, Tramu G. The role of vasoactive intestinal polypeptide in the inhibition of antral and pyloric electrical activity in rabbits. J Auton Nerv Syst. 1988;22(2):167-173.

38. Lu H, Zou Q, Gu H, Raichle ME, Stein EA, Yang Y. Rat brains also have a default mode network. Proc Natl Acad Sci. 2012;109(10):3979-3984.
39. Gertler R, Brown HC, Mitchell DH, Silvius EN. Dexmedetomidine: a novel sedative-analgesic agent. Baylor Univ Med Center Proc. 1999;75246:13-21.

40. Ailiani AC, Neuberger T, Brasseur JG, et al. Quantifying the effects of inactin vs Isoflurane anesthesia on gastrointestinal motility in rats using dynamic magnetic resonance imaging and spatio-temporal maps. Neurogastroenterol Motil. 2014;26(10):1477-1486.

41. Torjman MC, Joseph JI, Munsick C, Morishita M, Grunwald Z. Effects of Isoflurane on gastrointestinal motility after brief exposure in rats. Int J Pharm. 2005;294(1-2):65-71.

\section{SUPPORTING INFORMATION}

Additional supporting information may be found online in the Supporting Information section.

How to cite this article: Lu K-H, Cao J, Phillips R, Powley TL, Liu Z. Acute effects of vagus nerve stimulation parameters on gastric motility assessed with magnetic resonance imaging. Neurogastroenterol Motil. 2020;32:e13853.

https://doi.org/10.1111/nmo.13853 